\title{
Avaliação de cicatrizes cutâneas: apresentação de um método quantitativo
}

\author{
Assessment of surgical scars: a quantitative method
}

Fernando Mendonça Vidigal, TCBC-MG'; Andy Petroianu, TCBC-MG²

\section{R E S U M O}

\begin{abstract}
Objetivo: Descrever um método objetivo de avaliação das cicatrizes cutâneas com base em critérios quantificados e compará-lo com avaliação subjetiva do cirurgião e do paciente. Métodos: Foram analisados prospectivamente 136 pacientes submetidos à operações abdominais e inguinais. As cicatrizes foram avaliadas seis meses após o procedimento cirúrgico por meio da medida das alterações encontradas (alargamento, hipertrofia e retração). Essas cicatrizes foram classificadas em quatro tipos objetivos (A, B, C e D) e 10 subjetivos do cirurgião e do paciente (escore de 1 a 10). As classificações foram comparadas entre si. Resultados: $A$ avaliação subjetiva do cirurgião mostrou diferença entre os grupos 1 e $2(p=0,021)$ e 2 e 3B $(p=0,011)$. Na avaliação dos pacientes não houve diferença entre os grupos $(p=0,283)$. Em todos os grupos a avaliação do paciente foi mais satisfatória. A avaliação objetiva mostrou melhores resultados nos grupos 1 e 3B, sem diferença significativa. O método utilizado na avaliação objetiva foi eficaz e os valores médios atribuídos pelo cirurgião às cicatrizes dos tipos $A, B, C$ e D foram diferentes entre si $(p<0,001)$. Conclusão: A avaliação pelo método quantitativo foi adequada para classificar cicatrizes cirúrgicas. Esse método esteve mais de acordo com a realidade do que a impressão subjetiva apoiada em satisfação com o resultado obtido.
\end{abstract}

Descritores: Cicatriz. Técnicas de sutura.

\section{INTRODUÇÃO}

A investigação de cicatrizes cutâneas tem sido feita mediante diversos parâmetros - maioria deles com base em avaliações subjetivas. A diversidade dos métodos utilizados dificulta a comparação entre diferentes publicações e denota a ausência de um sistema aceito por todos os cirurgiões.

Parâmetros diversos têm sido empregados para classificar cicatrizes, desde valores específicos e graduados em escala numérica ${ }^{1-3}$, até subjetivos de conceituação ${ }^{4}$. A análise fotográfica comparativa é outro método amplamente utilizado, principalmente por cirurgiões plásticos $1,2,5,6$.

Um sistema de avaliação semi-quantitativa foi proposto por Trimbos e tem por base a conceituação do aspecto cicatricial de hipertrofia, cor e marcas transversais. A largura da cicatriz foi quantificada em milímetros, e o resultado final obtido pela soma dos escores das quatro alterações ${ }^{7}$.

Alguns autores adotaram uma escala visual, graduada em milímetros, para a pesquisa da satisfação do paciente ${ }^{5}$. Avaliação do aspecto estético da cicatriz no pósoperatório imediato foi utilizada por observadores médicos. Embora essa escala estética forneça compreensão sobre possíveis falhas na técnica de sutura, não é conhecida sua eficácia no pós-operatório recente ao correlacioná-la com a aparência tardia da cicatriz ${ }^{8}$. Comparação entre a satisfação do médico e do paciente com a aparência estética da cicatriz cutânea mostrou que existe ralação entre elas ${ }^{9}$.

Segundo Singer, em artigo de 1998, uma escala estética de feridas, para ser útil, precisa ser confiável, válida e clinicamente significante. Confiabilidade refere-se aos testes de consistência por medidas repetidas e é relativamente fácil de avaliar. Validade refere-se à maneira como a escala mede as características que são pretendidas no estudo. Deve ser instituída, de preferência, pela comparação dela com um critério padrão estabelecido. No caso de aparência estética de cicatrizes, nenhum critério padrão existe. Nessa situação, a nova escala pode ser validada pela comparação com outros testes que se propõem a medir as mesmas características. É preciso que a escala seja clinicamente aplicável, o que nem sempre implica em avaliação estatística. O resultado da classificação para ser considerado clinicamente válido deve se claramente percebido pelo paciente e pelo médico ${ }^{10}$.

Este trabalho descreve um método objetivo de investigação estética de cicatrizes cutâneas, com base em critérios quantitativos e de fácil aplicação clínica, que pode auxiliar na uniformização de conceitos, facilitando comparações, mediante parâmetros definidos.

Trabalho realizado na Santa Casa de Misericórdia de Juiz de Fora e Hospital Universitário da Universidade Federal de Juiz de Fora.

1. Professor Adjunto I do Departamento de Cirurgia da Faculdade de Medicina da Universidade Federal (UFMG); 2 . Professor Titular do Departamento de Cirurgia da Faculdade de Medicina da UFMG. 


\section{MÉTODOS}

Foram analisados prospectivamente 136 pacientes submetidos à operações para tratamento de afecções abdominais e inguinais. Foram excluídos os submetidos à operações contaminadas e infectadas; diabéticos; portadores de neoplasias avançadas; portadores de colagenoses; em uso prolongado de corticosteróides, imunossupressores ou anticoagulantes; aqueles com albumina sérica menor do que $3,1 \mathrm{~g} / \mathrm{dL}$ ou hemoglobina menor do que $10 \mathrm{~g} / \mathrm{dL}$; pacientes com cicatrizes pré-existentes, não retiradas completamente por ocasião da intervenção, no mesmo local da incisão cirúrgica.

De acordo com o tipo de fechamento da pele, os pacientes foram distribuídos aleatoriamente nos seguintes grupos:

Grupo 1 - síntese do tecido subcutâneo, sem sutura da pele com pontos separados de fio de categute simples 3-0 e nó invertido ou em " $U$ ", evitando a presença de espaço vazio. Em todos os casos, as bordas da pele foram aproximadas somente pelo fechamento do tecido subcutâneo, conforme preconizado por Petroianu em $1979{ }^{11}$.

Grupo 2 - sutura da pele com pontos simples transdérmicos utilizando fio de mononáilon preto 3-0, sem sutura do tecido subcutâneo;

Grupo 3- síntese da pele com sutura intradérmica contínua, sem sutura do tecido subcutâneo

Subgrupo $3^{\mathrm{a}}$ - com fio de mononáilon preto 3-0;

Subgrupo 3B - com fio de ácido poliglicólico bege

3-0.

As características dos grupos quanto ao sexo, idade e cor da pele são apresentadas na tabela 1. No Grupo 1 , as operações foram consideradas limpas em 21 (70 \%) pacientes e potencialmente contaminadas em nove (30\%). No Grupo 2, 23 (63,8 \%) operações foram consideradas limpas e $13(36,2 \%)$ potencialmente contaminadas. No Subgrupo 3A, as operações foram consideradas limpas em $26(66,7 \%)$ pacientes e potencialmente contaminadas em $13(33,3 \%)$ pacientes. No Subgrupo 3B, as operações fo- ram consideradas limpas em 17 (54,8 \%) pacientes e potencialmente contaminadas em 14 (45,2 \%).

Dentro da rotina pré-operatória para operações abdominais, a avaliação laboratorial incluiu hemograma, coagulograma, glicemia, creatinina e dosagem de proteínas séricas. Outros exames foram realizados de acordo com a necessidade dos doentes. Antibioticoprofilaxia foi instituída nos pacientes submetidos à operações potencialmente contaminadas e em casos específicos de operações limpas.

Todos os pacientes foram orientados a retornar para o primeiro controle entre o $12^{\circ}$ e o $15^{\circ}$ dias do pósoperatório, quando procedeu-se a remoção de pontos nos pacientes dos grupos 2 e 3A. O segundo controle foi realizado seis meses após o procedimento cirúrgico e teve por objetivo avaliar o resultado estético da cicatriz cirúrgica. A investigação constou de duas avaliações, uma subjetiva e outra objetiva.

\section{Avaliação subjetiva}

O cirurgião examinador atribuiu valor em uma escala de 1 a 10, orientado pelo aspecto estético da cicatriz cirúrgica, tomando-se como único critério sua aparência. O paciente também atribuiu valor em escala igual para a cicatriz, tendo por base o mesmo critério do cirurgião. Além disso, foi solicitado ao paciente que emitisse um conceito para a sua cicatriz (ótimo, bom, regular ou ruim).

\section{Avaliação objetiva}

Constou de inspeção e palpação da cicatriz, seguidas pela mensuração da sua parte mais larga, utilizando um paquímetro. As cicatrizes foram classificadas de acordo com o critério seguinte: 1) cicatriz normal - com menos de $2 \mathrm{~mm}$ de largura, que não apresentasse retração ou hipertrofia; 2) cicatriz alargada - alargamento igual ou superior a $2 \mathrm{~mm}$, subdividindo-se esse tipo em pequeno (2 $\mathrm{mm}$ a $3 \mathrm{~mm}$ de largura), intermediário (maior que $3 \mathrm{~mm}$ até $5 \mathrm{~mm}$ ) e grande (maior que $5 \mathrm{~mm}$ ); 3) cicatriz escavada - situada abaixo do nível da pele circunjacente e sua largura foi medida afastando-se as bordas, de modo a tor-

Tabela 1 - Características dos grupos quanto à identificação dos pacientes.

\begin{tabular}{|c|c|c|c|c|c|c|}
\hline \multirow[t]{3}{*}{$\begin{array}{l}\text { Grupo / } \\
\text { Subgrupo }\end{array}$} & \multirow[t]{3}{*}{$\begin{array}{c}2 \text { Idade } \\
(\mathrm{M} \pm \mathrm{DPM})\end{array}$} & \multicolumn{5}{|c|}{ Características } \\
\hline & & \multicolumn{2}{|c|}{ Sexo } & & \multicolumn{2}{|c|}{ Cor } \\
\hline & & $M$ & $F$ & $\mathrm{~L}$ & $F$ & $\mathrm{M}$ \\
\hline 1 & $45 \pm 18$ & 22 & 8 & 21 & 5 & 4 \\
\hline 2 & $45 \pm 15$ & 24 & 12 & 27 & 3 & 6 \\
\hline $3 \mathrm{~A}$ & $48 \pm 18$ & 28 & 11 & 28 & 0 & 11 \\
\hline $3 B$ & $47 \pm 16$ & 15 & 16 & 23 & 4 & 4 \\
\hline
\end{tabular}

Grupo -: síntese do tecido subcutâneo (fio de categute), sem sutura da pele.

Grupo 2 - sutura da pele com pontos simples (fio de mononáilon), sem sutura do tecido subcutâneo.

Subgrupo $3 A$ - síntese da pele com sutura intradérmica contínua, sem sutura do tecido subcutâneo (fio de mononáilon).

Subgrupo 3B - síntese da pele com sutura intradérmica contínua, sem sutura do tecido subcutâneo (fio de ácido poliglicólico).

Idade em anos.

Sexo: $m=$ masculino, $f=$ feminino.

Cor: L=leucodérmico, F: feodérmico, M: melanodérmico.

$M \pm$ DPM: média e desvio padrão da média. 
nar a cicatriz plana; 4) cicatriz hipertrófica - a que na palpação apresentasse consistência endurecida e elevando-se acima do nível da pele circunjacente e classificada quanto à sua largura em pequena (até $3 \mathrm{~mm}$ ), intermediária (maior que $3 \mathrm{~mm}$ até $5 \mathrm{~mm}$ ) e grande (maior que 5 $\mathrm{mm})$; 5) cicatriz quelóidea - a que crescesse fora dos limites originais da incisão cirúrgica.

De acordo com esses critérios, as cicatrizes foram classificadas em quatro tipos (Figuras 1, 2, 3 e 4): A normal - cicatriz de excelente qualidade; $B$ - hipertrofia pequena ou alargamento pequeno - cicatriz de boa qualidade; C - hipertrofia intermediária ou alargamento intermediário - cicatriz de qualidade regular; D - hipertrofia grande ou alargamento grande, ou quelóide - cicatriz de qualidade ruim.

Cicatrizes que tiveram alteração em um segmento igual ou menor a $20 \%$ de seu comprimento, independentemente do grau (pequeno, intermediário ou grande), foram classificadas como "B". Se a alteração ocorreu em extensão superior a $20 \%$ do seu comprimento, a classifi-

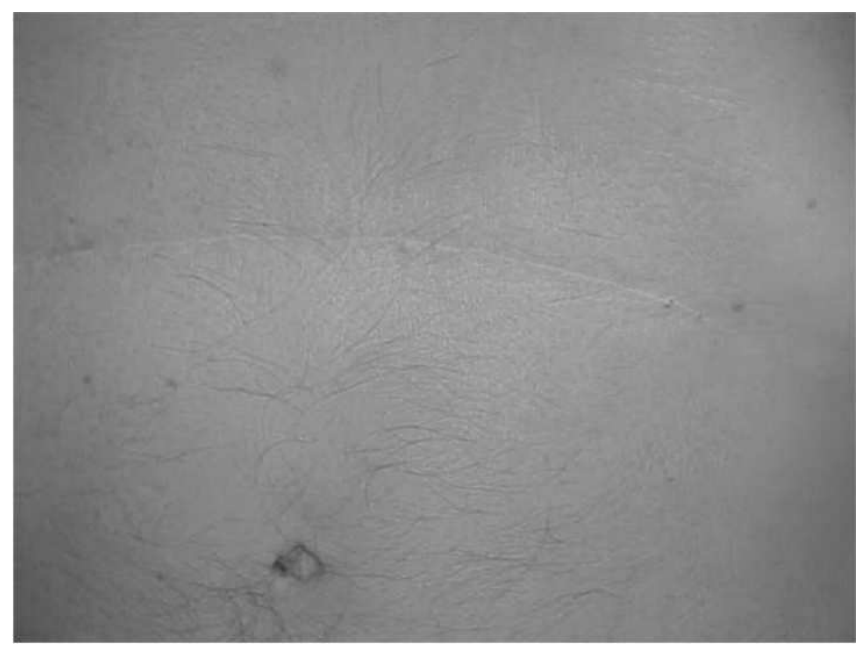

Figura 1 - Aspecto de cicatriz cirúrgica do tipo A (normal).

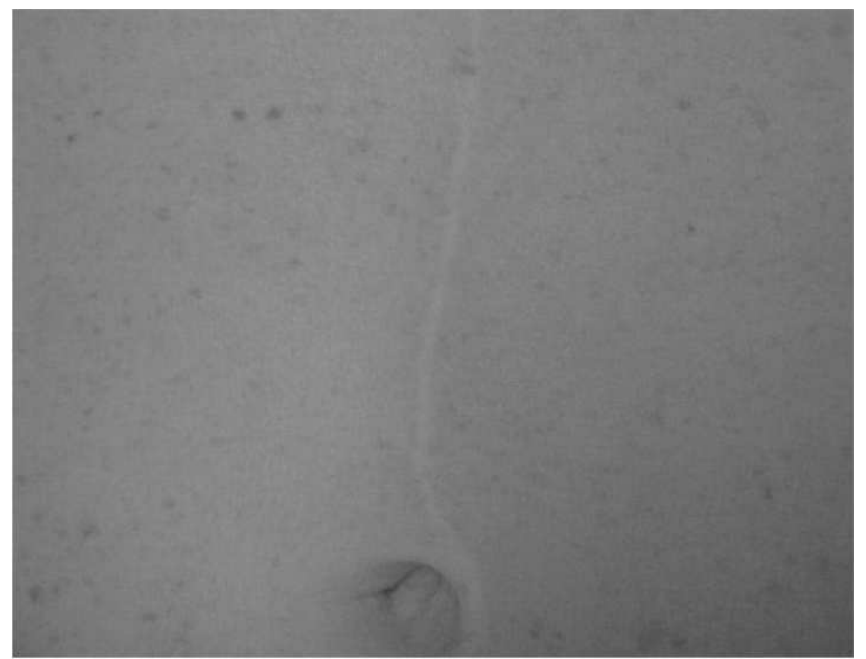

Figura 2 - Aspecto de cicatriz cirúrgica do tipo B (alargamento pequeno). cação seguiu os critérios já descritos. A que apresentou alargamento associado à hipertrofia foi classificada tomando como referência a alteração mais evidente ou de maior intensidade.

Foram realizados os testes qui-quadrado e exato de Fisher para determinar a associação entre os grupos estudados, além das variáveis sexo, idade, cor da pele e grau de contaminação. Com base nas proporções de cada grupo, no que se refere às complicações do segundo controle (hipertrofia e alargamento), foram realizados testes para diferença de duas proporções, comparando os grupos dois a dois. Nas avaliações subjetivas e objetiva, foram realizados os testes $t$ de Student, Mann Whitney, ANOVA, LSD e Kruskall Wallis. O mesmo foi feito para comparação entre os valores atribuídos pelos pacientes e pelo examinador, tendo como referência a classificação objetiva ( $A, B, C$ e D). O nível de significância adotado foi de $5 \%$ e os cálculos foram realizados com o auxílio do programa estatístico SPSS - Statistical Package for Social Sciences v.11.01.

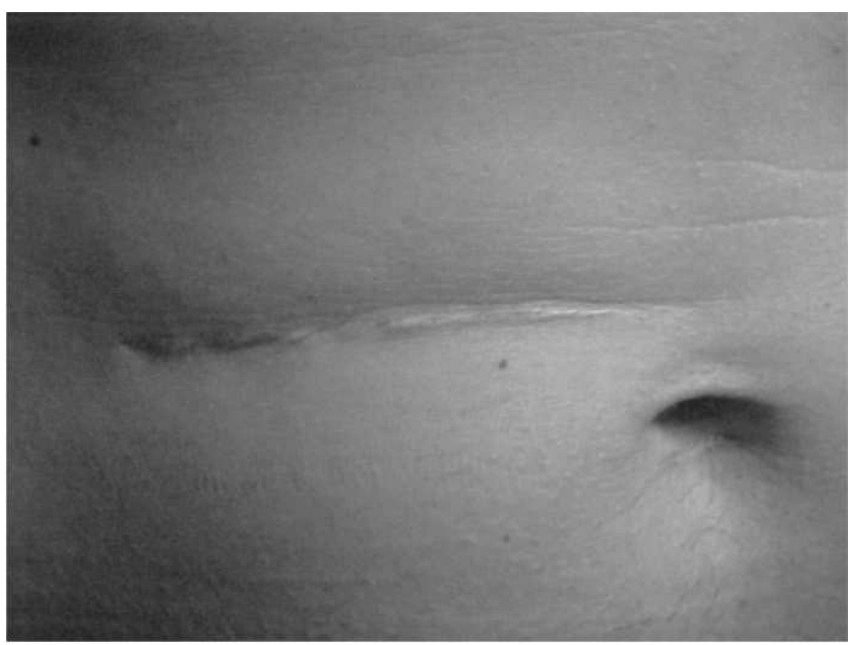

Figura 3 - $\quad$ Aspecto de cicatriz cirúrgica do tipo C (alargamento intermediário).

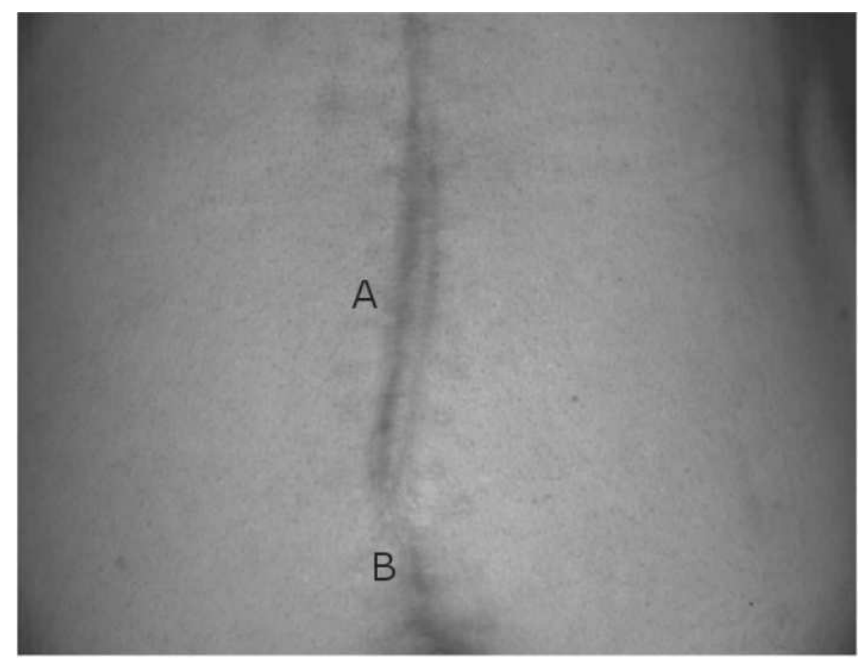

Figura 4 - $\quad$ Aspecto da cicatriz cirúrgica do tipo D. Observa-se hipertrofia grande em $A$ e alargamento intermediário em $B$. 


\section{RESULTADOS}

Os grupos foram comparados pelo teste do quiquadrado, em relação ao sexo $(p=0,137)$, idade $(p=0,221)$, cor da pele $(p=0,161)$ e grau de contaminação da operação $(p=0,633)$. Não houve diferenças entre as características dos pacientes e das operações nos quatro grupos analisados.

Vinte e seis não compareceram para o segundo controle, restando 113, assim distribuídos: 26 no Grupo 1; 28 no Grupo 2; 32 no Subgrupo 3a; 27 no Subgrupo 3B. No Grupo 1, o tempo médio para o comparecimento foi de $236 \pm 63$ dias pós-operatórios; no Grupo 2, $220 \pm 91$ dias; no Subgrupo 3A, $227 \pm 72$ dias; e no Subgrupo 3B, $205 \pm$ 41 dias, valores esses sem diferenças entre si $(p=0,443)$.

\section{da cicatriz}

Avaliação subjetiva do aspecto estético

A avaliação subjetiva feita pelo cirurgião examinador não foi difícil; no entanto, muitos pacientes tiveram dificuldade em atribuir um valor, em escala de 1 a 10, para classificar a sua cicatriz. Um doente não conseguiu sequer atribuí-lo. Porém, quando solicitados a conceituar a cicatriz (ótima, boa, regular ou ruim), todos o fizeram sem dificuldade e a maioria ficou satisfeita com o resultado estético final, independentemente do grupo.

No Grupo 1 a avaliação do cirurgião examinador resultou em valor médio de 8,0, em escala de 1 a 10, enquanto na opinião do paciente foi de 9,1. Houve diferença entre as duas avaliações $(p=0,012)$. Solicitados a emitir opinião sobre a qualidade da cicatriz, quatro pacientes consideraram-na ótima, 20, boa, um, regular e um, ruim (Tabela 2).

No Grupo 2 o valor médio atribuído pelo cirurgião examinador às cicatrizes dos pacientes deste grupo foi de 6,9, enquanto a média dos valores atribuídos pelos pacientes foi de 8,6. Houve diferença entre as duas avaliações $(p<0,001)$. Além disso, quatro pacientes consideraram a cicatriz ótima, 20 consideraram-na boa e quatro, ruim (Tabela 2 ).

No Subgrupo 3A a média dos valores atribuídos pelo cirurgião examinador foi de 7,5, enquanto a dos pacientes foi de 9,5. Houve diferença entre as duas avaliações $(p<0,001)$. Cinco pacientes conceituaram a cicatriz como ótima, 25 consideraram-na boa, um, regular, e um, ruim (Tabela 2).

No Subgrupo 3B a média dos valores na avaliação do cirurgião examinador foi de 8,1 e a dos pacientes foi de 9,4 . Houve diferença entre as duas avaliações $(p=$ 0,001 ). Três pacientes consideraram a cicatriz ótima, 21 consideraram-na boa, dois, regular e um, ruim. Um paciente não conseguiu atribuir um valor para sua cicatriz. (Tabela 2).

\section{Comparação entre os grupos}

Foram comparados os valores médios atribuídos pelo examinador por grupos, por meio do teste ANOVA. A diferença foi significativa $(p=0,041)$ e ocorreu entre os grupos 1 e 2 ( $p=0,021)$, e Grupo 2 e Subgrupo 3B ( $p=$ $0,011)$.

Os valores médios atribuídos pelos pacientes foram comparados entre os grupos, não havendo diferença entre eles $(p=0,283)$. Em todos os grupos a avaliação do paciente foi mais satisfatória que a do cirurgião.

\section{cicatriz}

\section{Avaliação objetiva do aspecto estético da}

No Grupo 1 as cicatrizes normais ocorreram em nove pacientes, oito tiveram cicatriz hipertrófica e sete tiveram cicatriz alargada. Em dois pacientes foram registradas

Tabela 2 - Avaliação subjetiva do aspecto estético da cicatriz após período superior a seis meses.

\begin{tabular}{|c|c|c|c|c|c|c|c|c|c|c|}
\hline \multirow[t]{3}{*}{ Avaliação } & \multicolumn{8}{|c|}{ Grupo/Subgrupo } & \multicolumn{2}{|c|}{ Total } \\
\hline & \multirow{2}{*}{\multicolumn{2}{|c|}{$\begin{array}{c}1 \\
26 \\
\text { n (\%) }\end{array}$}} & \multirow{2}{*}{\multicolumn{2}{|c|}{$\begin{array}{c}2 \\
28 \\
\text { n (\%) }\end{array}$}} & \multirow{2}{*}{\multicolumn{2}{|c|}{$\begin{array}{c}3 \mathrm{~A} \\
32 \\
\mathrm{n}(\%)\end{array}$}} & \multirow{2}{*}{\multicolumn{2}{|c|}{$\begin{array}{c}3 \mathrm{~B} \\
27 \\
\mathrm{n}(\%)\end{array}$}} & \multirow{2}{*}{\multicolumn{2}{|c|}{$\begin{array}{c}113 \\
\mathrm{n}(\%)\end{array}$}} \\
\hline & & & & & & & & & & \\
\hline Ótima & 4 & $(15,4)$ & 4 & $(14,3)$ & 5 & $(15,6)$ & 3 & $(11,1)$ & 16 & $(14,2)$ \\
\hline Boa & 20 & $(76,9)$ & 20 & $(71,4)$ & 25 & $(78,1)$ & 21 & $(77,8)$ & 86 & $(76,1)$ \\
\hline Regular & 1 & $(3,8)$ & - & & 1 & $(3,1)$ & 2 & $(7,4)$ & 4 & $(3,5)$ \\
\hline Ruim & 1 & $(3,8)$ & & $(14,3)$ & 1 & $(3,1)$ & 1 & $(3,7)$ & 7 & $(6,2)$ \\
\hline \multicolumn{11}{|l|}{ Valor/cirurgião } \\
\hline $\begin{array}{l}\mathrm{M} \pm \mathrm{DPM} \\
\text { Valor/paciente }\end{array}$ & \multicolumn{2}{|c|}{$8 \pm 1,6$} & \multicolumn{2}{|c|}{$6,9 \pm 1,6$} & \multicolumn{2}{|c|}{$7,5 \pm 1,6$} & \multicolumn{2}{|c|}{$8,1 \pm 1,6$} & & \\
\hline $\mathrm{M} \pm \mathrm{DPM}$ & \multicolumn{2}{|c|}{$9,1 \pm 1,7$} & \multicolumn{2}{|c|}{$8,6 \pm 2,4$} & \multicolumn{2}{|c|}{$9,5 \pm 1,2$} & \multicolumn{2}{|c|}{$9,4 \pm 1,2$} & & \\
\hline
\end{tabular}

Grupo 1: síntese do tecido subcutâneo (fio de categute), sem sutura da pele.

Grupo 2: sutura da pele com pontos simples (fio de mononáilon), sem sutura do tecido subcutâneo.

Subgrupo 3A: síntese da pele com sutura intradérmica contínua, sem sutura do tecido subcutâneo (fio de mononáilon).

Subgrupo 3B: síntese da pele com sutura intradérmica contínua, sem sutura do tecido subcutâneo (fio de ácido poliglicólico).

n: número de pacientes

$\%$ : porcentagem

M: média

DPM: desvio padrão da média

(-): avaliação não observada 
cicatrizes mistas. Alargamento associado à hipertrofia ocorreu em um caso e hipertrofia associada a retração em outro. Quatro pacientes não compareceram para o controle. Com base na avaliação quantitativa das alterações cicatriciais, houve nove cicatrizes do tipo $A$, sete do tipo $B$, cinco do tipo $\mathrm{C}$ e cinco do tipo D (Tabela 3).

No Grupo 2, seis pacientes tiveram cicatrizes normais e treze largas, sendo três com retração parcial conferindo-lhe aspecto escavado. Cicatrizes hipertróficas ocorreram em cinco pacientes, sendo que em quatro deles houve associação com alargamento cicatricial. Não compareceram para o controle oito pacientes.

Foram observadas cicatrizes transversais evidentes em dez pacientes, comprometendo o aspecto estético. Em quatro casos as cicatrizes transversais eram finas.

A classificação quantitativa das alterações cicatriciais foram encontradas em seis cicatrizes do tipo $A$, sete do tipo $B$, oito do tipo $C$ e sete do tipo D (Tabela 3 ).

No Subgrupo 3A foram registrados cinco pacientes com cicatrizes normais, alargamento cicatricial ocorreu em 14 pacientes e sete tiveram hipertrofia da cicatriz. Em seis, houve associação de hipertrofia e alargamento, sendo que um deles apresentou também retração em parte da cicatriz. Não compareceram para o controle sete pacientes.

A classificação com base na quantificação das alterações cicatriciais resultou em cinco cicatrizes do tipo A, 11 do tipo B, cinco do tipo C e 11 do tipo D (Tabela 3).

No Subgrupo 3B foram verificadas sete cicatrizes normais, nove com alargamento e oito com hipertrofia. Em três casos, houve associação de alargamento e hipertrofia. Não compareceram para o controle quatro pacientes.

A classificação com base na quantificação das alterações cicatriciais resultou em sete cicatrizes do tipo $A$, 11 do tipo $B$, cinco do tipo $C$ e quatro do tipo D (Tabela 3 ).

Comparação entre as avaliações objetiva e subjetiva das cicatrizes.
A comparação entre as avaliações objetiva e subjetiva do cirurgião examinador evidenciou diferença entre as médias dos valores atribuídos para as cicatrizes dos tipos $A, B, C$ e D $(p<0,001)$. Na avaliação subjetiva do paciente, a média dos valores atribuídos para as cicatrizes dos tipos $A, B$ e $C$ foram semelhantes. Os valores atribuídos às do tipo $D$ foram diferentes em relação aos tipos $A, B$ e $C$ ( $p$ $<0,001, p=0,001$ e $p=0,01$, respectivamente).

Comparando as médias dos valores atribuídos pelo cirurgião examinador com as do paciente, observou-se diferença para as cicatrizes dos tipos $B, C$ e $D(p<0,001)$. Não houve diferença para a cicatriz do tipo $A(p=0,126)$ (Figura 5).

\section{DISCUSSÃO}

O controle tardio da cicatriz cirúrgica, quanto ao aspecto estético, acompanha-se de opiniões divergentes,

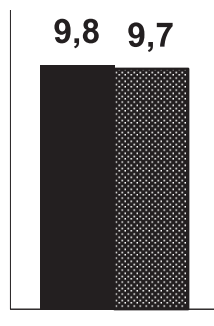

A

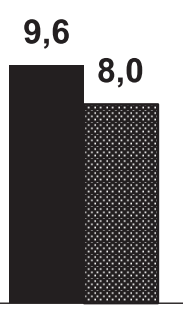

B
9,4

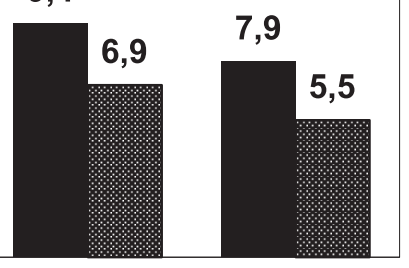

C

\section{Tipos de cicatriz}

Figura 5 - $\quad$ Média dos valores conferidos pelo cirurgião examinador e pelo paciente nos quatro tipos de cicatrizes.

Tabela 3 - Incidência dos tipos de cicatrizes nos grupos 1, 2, 3A e 3B.

\begin{tabular}{|c|c|c|c|c|c|c|c|c|c|c|}
\hline \multirow[t]{4}{*}{ Cicatriz } & \multicolumn{8}{|c|}{ Grupo/Subgrupo } & \multicolumn{2}{|c|}{ Total } \\
\hline & \multicolumn{2}{|c|}{1} & \multicolumn{2}{|r|}{2} & \multicolumn{4}{|c|}{3} & \multirow{3}{*}{\multicolumn{2}{|c|}{$\begin{array}{c}113 \\
\text { n (\%) }\end{array}$}} \\
\hline & \multirow{2}{*}{\multicolumn{2}{|c|}{$\begin{array}{c}26 \\
n(\%)\end{array}$}} & \multirow{2}{*}{\multicolumn{2}{|c|}{$\begin{array}{c}28 \\
n(\%)\end{array}$}} & \multicolumn{2}{|c|}{$A$} & \multicolumn{2}{|c|}{ B } & & \\
\hline & & & & & \multicolumn{2}{|c|}{$\begin{array}{c}32 \\
n(\%)\end{array}$} & \multicolumn{2}{|c|}{$\begin{array}{c}27 \\
n(\%)\end{array}$} & & \\
\hline A & 9 & $(34,6)$ & 6 & $(21,4)$ & 5 & $(15,6)$ & 7 & $(25,9)$ & 27 & $(23,9)$ \\
\hline B & 7 & $(26,9)$ & 7 & $(25)$ & 11 & $(34,4)$ & 11 & $(40,7)$ & 36 & $(31,9)$ \\
\hline C & 5 & $(19,2)$ & 8 & $(28,6)$ & 5 & $(15,6)$ & 5 & $(18,5)$ & 23 & $(20,4)$ \\
\hline D & 5 & $(19,2)$ & 7 & $(25)$ & 11 & $(34,4)$ & 4 & $(14,8)$ & 27 & $(23,9)$ \\
\hline
\end{tabular}

(A): Normal. Cicatriz de excelente qualidade.

(B): Hipertrofia pequena ou alargamento pequeno. Cicatriz de boa qualidade.

(C): Hipertrofia intermediária ou alargamento intermediário. Cicatriz de qualidade regular.

(D): Hipertrofia grande ou alargamento grande, ou quelóide. Cicatriz de qualidade ruim.

Grupo 1: síntese do tecido subcutâneo (fio de categute), sem sutura da pele.

Grupo 2: sutura da pele com pontos simples (fio de mononáilon), sem sutura do tecido subcutâneo.

Grupo 3A: síntese da pele com sutura intradérmica contínua, sem sutura do tecido subcutâneo (fio de mononáilon).

Subgrupo 3B: síntese da pele com sutura intradérmica contínua, sem sutura do tecido subcutâneo (fio de ácido poliglicólico).

n: número

$\%:$ porcentagem 
tanto em relação ao momento ideal de estudo, quanto aos critérios utilizados na avaliação. Alguns autores sugerem que a avaliação estética da cicatriz deva ser feita após um ano, com base no fato de que o colágeno está totalmente maturado e a ferida completamente cicatrizada ${ }^{12}$. Outros autores têm avaliado a cicatriz após seis meses ${ }^{13-15}$. Segundo Trimbos et al., a avaliação da cicatriz com seis meses pós-operatórios é tão confiável quanto com um ano ${ }^{16}$. Há ainda cirurgiões que consideram suficiente a revisão após três meses ${ }^{12,17}$. No período de três meses, a ferida tem 80 $\%$ da resistência original da pele, sendo improvável que alterações significativas em sua remodelação ou seu alargamento ocorram após esse tempo, modificando a evolução estética ${ }^{12}$, a menos que ocorra interferência externa ou se forme quelóide, fatos esses que não foram encontradas neste trabalho.

Na presente casuística, optou-se pela revisão da cicatriz com seis meses pós-operatórios, tendo por base a literatura ${ }^{13-15}$. Ressalta-se, ainda, que o acompanhamento de pacientes no pós-operatório por longo prazo é difícil, pelo baixo índice de comparecimento nos controles mais tardios, se o paciente não tiver queixas relacionadas à operação.

A ausência de um método objetivo de avaliação clínica torna o estudo da cicatriz um problema complexo e de conceituação difícil. As avaliações subjetivas, tanto de pacientes quanto de examinadores, são imprecisas e utilizam parâmetros distintos, dificultando a comparação de resultados. A aparência estética é o método mais apropriado para avaliar a evolução da síntese de uma ferida cutânea. No entanto, a subjetividade dessa avaliação leva à imprecisão de conceitos, mesmo entre médicos de mesma especialidade e experiência. A avaliação pelos pacientes poderia ser influenciada pelo sexo, idade, condições socioeconômicas e culturais, além de crenças religiosas e relacionamento com o cirurgião. Portanto, a comparação de resultados entre diferentes populações torna-se muito difícil. Um sistema objetivo, mensurável e de fácil aplicação clínica, que complemente a avaliação subjetiva, é fundamental para a quantificação de alterações da cicatrização. A avaliação objetiva utilizada no presente estudo atendeu a esses quesitos.

Para a avaliação subjetiva do cirurgião e do paciente, optou-se pela atribuição de valores em uma escala de 1 a 10, método já utilizado tanto para pacientes ${ }^{2}$ quanto para examinadores independentes'. Comparar essas opiniões é válido, principalmente por ter sido pouco relatado na literatura.

Os pacientes tiveram, por vezes, dificuldade em associar um valor numérico à qualidade estética da cicatriz. Em geral, os valores atribuídos por eles foram elevados, indistintamente em todos os grupos, independentemente do seu aspecto, o que sugere incapacidade de discernimento entre as cicatrizes ou cordialidade com o cirurgião. Quando solicitada a conceituar a cicatriz, a maioria dos doentes considerou-a de boa qualidade e mostrouse satisfeita com ela.

É provável que o aspecto estético tenha sido considerado secundário, indicando que a cicatriz representava muito mais a solução da afecção que levou ao tratamento cirúrgico do que uma sequela. Esses resultados não devem ser extrapolados para outras populações com cultura e costumes diferentes, principalmente por utilizar critérios subjetivos. Cabe, dessa maneira, associar uma classificação com base em critérios quantificados isentos de influências imponderáveis.

A avaliação do cirurgião examinador encontrou resultados menos favoráveis que a do paciente em todos os aspectos, com valores médios mais baixos em todos os grupos. As avaliações objetiva e subjetiva do cirurgião foram coerentes. Os valores atribuídos para as cicatrizes dos tipos A, B, C e D denotaram distinção evidente entre elas. $O$ mesmo não ocorreu na avaliação dos pacientes, que não diferenciaram bem as cicatrizes, atribuindo valores semelhantes para os tipos A, B e C. Apenas nas cicatrizes do tipo $D$ os valores foram menores. O sistema de avaliação objetiva utilizado neste trabalho parece ser confiável e está de acordo com a avaliação subjetiva do cirurgião, permitindo classificação compreensível do aspecto estético da cicatriz cirúrgica. No entanto, apesar dessa impressão inicial, há a necessidade de sua utilização em outros trabalhos científicos para comprovar sua real eficácia clínica.

Em conclusão, os resultados desse trabalho mostram que é possível quantificar a avaliação do aspecto estético da cicatriz e sugerem que o paciente tende a ficar mais satisfeito do que o cirurgião com cicatrizes abdominais esteticamente imperfeitas.

\section{A B S T R A C T}

\footnotetext{
Objective: To describe an objective assessment method, based on quantifiable criteria, and compare it with subjective patient and surgeon assessments. Methods: 136 patients undergoing abdominal surgery were prospectively analyzed. Surgical scars were evaluated six months after surgery, with measurements of widening, hypertrophy and retraction. An objective 4-type scar classification ( $A, B, C$ and $D)$ and a subjective 10-point surgeon and patient-assessed score were used. The results were compared. Results: The surgeon's subjective assessment was different between groups 1 and $2(p=0.021)$ and between groups 2 and $3 B(p=0.011)$. Patients' assessments did not significantly differ between the groups $(p=0.283)$. The objective assessment gave better results for groups 1 and $3 B$, although without significance. The method used for objective assessment was effective and the surgeon's mean scores differed between the groups $(p<0.001)$. Conclusion: Assessment using the quantitative method was adequate to classify surgical scars, and agreed more with reality than a satisfaction-based subjective impression.
}

Key words: Cicatrix. Suture techniques. 


\section{REFERENCIAS}

1. Lubowski D, Hunt D. Abdominal wound closure comparing the Proximate stapler with sutures. Aust N Z J Surg. 1985; 55(4):4056.

2. Sakka AS, Graham AK, Abdulah A. Skin closure in hip surgery: subcuticular versus transdermal a prospective randomized study. Acta Orthop Belg. 1995; 61(4):331-6.

3. Risnes I, Abdelnoor M, Baksaas ST, Lundblad R, Svennevig JL. Sternal wound infections in patients undergoing open heart surgery: randomized study comparing intracutaneous an transcutaneous suture techniques. Ann Thorac Surg. 2001; 72(5):1587-91.

4. Pedersen VM, Jensen BS, Hansen B. Skin closure in abdominal incisions. Continuous nylon suture versus Steristrip tapesuture: a controlled trial. Acta Chir Scand. 1981; 147(8):619-22.

5. Quinn JV, Drzewiecki A, Li MM, Stiell EG, Sutcliffe T, Elmslie TJ. Appearance scales to measure cosmetic outcomes of healed lacerations. Am J Emerg Med. 1995; 13(2):229-31.

6. Beausang $\mathrm{E}$, Floyd $\mathrm{H}$, Dunn, KW, Orton Cl, Ferguson MWJ. A new quantitative scale for clinical scar assessment. Plast Reconstr Surg. 1998; 102(6):1954-61.

7. Trimbos JB, Smeets $M$, Verdel M, Hermans J. Cosmetic result of lower midline laparotomy wounds: polibutester and nylon skin suture in a randomized clinical trial. Obstet Gynecol. 1993; 82(3):390-3.

8. Hollander JE, Singer AJ, Valentine, S, Henry, M. Wound registry: development and validation. Ann Emerg Med. 1995; 25(5):67585. Erratum in: Ann Emerg Med 1995; 26(4):532

9. Singer AJ, Church AL, Forrestal K, Werblud M, Valentine, SM, Hollander JE. Comparison of patient satisfaction and practitioner satisfaction with appearance after traumatic wound repair. Acad Emerg Med. 1997; 4(2):133-7.

10. Singer AJ. Clinical wound evaluation scales. Acad Emerg Med. 1998; 5(6):564-6.
11. Petroianu A. Synthesis of surgical wounds whitout skin suture Sir. Plast Reconstr Surg. 1988; 82(5):919.

12. Guyuron B, Vaughan C. A comparison of absorbable and nonabsorbable suture materials for skin repair. Plast Reconstr Surg. 1992; 89(2):234-6.

13. Shetty PC, Dicksheet S, Scalea TM. Emergency department repair of hand lacerations using absorbable Vicryl sutures. J Emerg Med. 1997; 12(5):763-4.

14. Gabel EA, Jimenez GP, Eaglstein WH, Kerdel FA, Falanga $V$. Performance comparison of nylon and an absorbable suture material (polyglactin 910) in the closure punch biopsy sites. Dermatol Surg. 2000; 26(8):750-2; discussion 752-3.

15. Chen HH, Tsai WS, Yeh CY, Wang JY, Tang R. Prospective study comparing wounds closed with tape with suture interrupted wounds in colorectal surgery. Arch Surg. 2001; 136(7):801-3.

16. Trimbos JB, Mouw R, Ranke G, Trimbos KB, Zwinderman K. The Donati stitch revisited: favorable cosmetic results in a randomized clinical trial. J Surg Res. 2002; 107(1):131-4.

17. Soisson AP, Olt G, Soper JT, Berchuck A, Rodrigues G, Clarke-Pearson $\mathrm{DL}$. Prevention of superficial wound separation with subcutaneous retention sutures. Gynecol Oncol. 1993; 51(3):330-4.

Recebido em 23/01/2009

Aceito para publicação em 24/03/2009

Conflito de interesse: nenhum

Fonte de financiamento: nenhuma

\section{Como citar esse artigo:}

Vidigal FM, Petroianu A. Avaliação de cicatrizes cutâneas: apresentação de um método quantitativo. Rev Col Bras Cir. [periódico na Internet] 2010; 37(2). Disponível em URL: http://www.scielo.br/rcbc

\section{Endereço para correspondência:}

Andy Petroianu

E-mail: petroian@gmail.com 